\title{
Ein „haymatloser“ Übersetzer in der Türkei: Cornelius Bischoff
}

\author{
Müge Arslan Karabulut (D), Konya
}

https://dx.doi.org/10.37583/diyalog.802275

\begin{abstract}
Deutsch)
Die Übersetzung, insbesondere die literarische, ist vor allem eine Art Kulturübertragung. Neben der Beherrschung der Sprachen setzt sie die Kenntnis des Allgemeinen und Besonderen des Landes wie Kultur, Tradition, Glauben, geschichtliche und gesellschaftliche Begebenheiten und auch soziale Strukturen voraus. Wenn die Sprachen und Kulturen tiefgreifend wahrgenommen werden, können die übersetzten Texte die Adressaten erreichen, d.h., dass die Ausgangssprache und -kultur für die Zielrezipienten verständlich sein können. So wird der Übersetzer als Kulturträger angenommen. Cornelius Bischoff ist beispielsweise ein wohlbekannter Name für den deutschen und türkischen Literaturkreis. Er ist vor allem bekannt als „der deutscheste Türke und der türkischste Deutsche“ sowie als eine Brücke zwischen Deutschland und der Türkei.

Das Ziel der vorliegenden Arbeit ist es, Cornelius Bischoff, einen Kulturträger zwischen der deutschen und türkischen Übersetzung, zu behandeln. Als ein „Haymatloser“ fand er in der Türkei die Möglichkeit, die türkische Sprache und Kultur wesentlich kennenzulernen und viele türkische Werke ins Deutsche zu übersetzen. Als ein Übersetzer trug er zuallererst dazu bei, die türkische Literatur, die bedeutenden türkischen Schriftsteller, die türkische Kultur und Tradition sowie den türkischen Sprachgebrauch in Deutschland bekannt zu machen. In der vorliegenden Arbeit wird die Besonderheit Bischoffs in der Übersetzungswelt im Hinblick auf drei Aspekte diskutiert: zuerst im Hinblick auf den Zusammenhang seiner Wurzeln in der Türkei und im Türkischen -schon in seinen Wurzeln, besonders mütterlicherseits, wurde das Türkische verinnerlicht-, dann auf die in der Türkei verbrachten Jahre -die Jahre, in denen er "haymatlos“ genannt wurde- und zuletzt auf die Wahrnehmung und Aneignung der türkischen Sprache, Kultur und Gesellschaft - was auf ihn lebenslang einwirkte-. In diesem Kontext wird versucht, sein Leben, seine Werke und seine Wirkung im Rahmen der übersetzerischen Tätigkeit zu analysieren.
\end{abstract}

Schlüsselwörter: Literarische Übersetzung, Cornelius Bischoff, haymatlos, das Deutsche, das Türkische.

\section{Abstract (English) \\ A "haymatlos" Translator in Turkey: Cornelius Bischoff}

The translation, especially the literary translation, is above all a kind of cultural transfer. In addition to knowing languages, it requires knowledge of the general and in the particular, of the languages, its culture, tradition, and belief, history and also social structure. Only then the translated text can reach the recipients, enable the source language and culture. So, it is understandable for the target language and culture, and by doing so, the translator can be accepted as a cultural carrier if both language and culture are deeply perceived. The German and Turkish literary circles know such a translator: Cornelius Bischoff. 
He is first of all famous as "the most German of the Turks and the most Turk of the Germans" and as a bridge between Germany and Turkey.

The aim of the present work is to treat Cornelius Bischoff as culture-bearer between the German and Turkish translation. As a "haymatlos" in Turkey, he had the opportunity to get to know the Turkish language and culture substantially and then transfers so many important Turkish works to German. As a translator, he contributed to making Turkish literature, the important Turkish writers, Turkish culture and tradition as well as the Turkish usage known in Germany. In the present work, the speciality of Bischoff in the field of translation is discussed with regard to the following three aspects: first with the connection between his roots in Turkey and in Turkish - Turkish was already internalized in its roots, especially on his mother's side -, then with regard to years in Turkey - the years in which he was called "haymatlos" and finally with regard to the perception of the Turkish language, culture and society - which lasted for a lifetime for him. In this context, an attempt is made to analyze his life, work and effect in the context of the field of translation.

Keywords: Literary translation, Cornelius Bischoff, haymatlos, German, Turkish. 


\section{EXTENDED ABSTRACT}

Culture, history, and social structure are the most important components of a language, both in factual and literary texts. In this way, every text comes about as a result of the interaction between culture and language. In this regard, when translating, it is necessary to have a good knowledge of the culture, history, speech formation style of feelings and thoughts as well as the linguistic structure and the particularity of the language used in the source language (including the author). The translator should be familiar with the general aesthetic and literary usage and take them into account during the translation process. The translation of literary works brings the idea of world literature even closer, and the translator assumes a special role in the recognition of literature in the other languages.

The close Turkish-German relationship also affects translation. As a result of this relationship, interest in literature and the literary transfers of the foreign culture and language has increased. Before 1923 this interest was mainly limited to the military sector, but after 1923 the number of literary translations from German into Turkish or vice versa increased, in order to get to know the culture of the foreigner. In this regard, cultural transmission became more important. This gave those translators an advantage who not only mastered both languages but also both cultures.

Cornelius Bischoff was a translator who played an important role in the recognition of Turkish literature in Germany. He had succeeded in becoming known as a carrier of culture between the Turkish and the German. Bischoff's main goal when translating was to bring the German and Turkish language and culture together and to ensure that the German reader gets to know the Turkish culture and language or Turkey properly and thoroughly.

In the given paper, the life, works and impact of Bischoff in the context of his translations from Turkish into German are analyzed, especially with regard to his experiences in Turkey. Bischoff's role in translation is generally viewed in terms of the connection between his roots in Turkey and Turkish, the years spent in Turkey and the perception of the Turkish language, culture and society.

Bischoff was born in Hamburg-Harburg in 1928 as the son of a German carpenter and a Jewish woman of Serbian descent who had lived in Istanbul for a long time. In 1939 he and his family fled to Istanbul from the Nazi regime. From 1944 to 1945 he was interned with his family as a German citizen in the Anatolian Çorum. They were interned in Çorum with the stamp "Haymatloz" on their identity card. The statement "a haymatlos translator" in this work refers first to the years of Bischoff's life in Turkey. It is also believed that Bischoff had a good command of the Turkish language and culture as well as German. So "being haymatlos" here does not indicate a deficiency as something negative, but rather to Bischoff's plurality.

The connection between Bischoff and Turkey is mainly due to the fact that his mother had a close connection with Turkey and that Bischoff himself lived in Turkey for a long time. He did not study Turkology, but learned the Turkish language and culture in Turkey. Bischoff always considered his relationship with Turkey to be 
something special and different from other translators. His bicultural and bilingual life formed a unit. He was equally at home in both languages and cultures. There are no borders for him between German and Turkish and between Turkey and Germany.

Bischoff's translation technique was reproductive, which showed his creativity. He grasped and interpreted the Turkish language and culture as a person of German origin who lived in Turkey for a long time, from a foreign and local perspective. In this regard, he was able to thoroughly analyze, interpret, and then translate literary texts within the framework of form and content. He was privileged because of the opportunity to experience the general and the specific features of Turkish in Turkey. 


\section{Einleitung}

Das Übersetzen hat neben der Beherrschung der Sprachen zuallererst mit der Beherrschung der mehrdimensionalen Werte wie „Weltwissen, Kulturwissen, Allgemeinbildung, die Bekanntschaft mit Gebräuchen, Sitten, Traditionen anderer Gemeinschaften, unterschiedliche Weltanschauungen“" zu tun (Uyanık 2019: vii). Die Kultur, Geschichte und gesellschaftliche Struktur veräußerlichen sich als die wichtigsten Bestandteile der Sprache sowohl in sachlichen als auch in literarischen Texten. Auf diese Weise kommt jeder Text infolge der Wechselwirkung zwischen der Kultur und Sprache zustande.

Im Vergleich $\mathrm{zu}$ anderen Textsorten enthalten literarische Texte mehrere subjektive, gesellschaftliche, geschichtliche, kulturelle oder auch politische Eigenschaften. Sie dienen nicht nur zur Übertragung der Regeln der Standartsprache, sondern auch der Umgangssprache. Sie tragen somit sowohl zur Sprachübertragung als auch zur Kulturübertragung bei (vgl. Uyanı/ Uslu 2013: 1408ff). In dieser Hinsicht ist es bei der Übersetzung erforderlich, die Kultur, Geschichte, Gestaltungsweise der Gefühle und Gedanken sowie die linguistische Struktur und die Besonderheit des Sprachgebrauchs der Ausgangssprache (auch des Autors) gut zu kennen. Der Übersetzer soll mit dem allgemeinen ästhetischen und literarischen Sprachgebrauch vertraut sein und diesen beim Übersetzungsprozess berücksichtigen.

Die literarische Übersetzung hat eine wichtige Rolle zwischen den Sprachen und Kulturen. Ausgehend von übersetzten Werken kann man sowohl die Sprache, die Kultur und die Autoren als auch die gesellschaftlichen, psychologischen, politischen, historischen und philosophischen Begebenheiten des „Anderen“ (der ,anderen“ Sprachen und Kulturen) kennenlernen. Durch die Übersetzung von literarischen Werken wird nämlich dem Gedanken an die Weltliteratur noch nähergekommen, und der Übersetzer übernimmt eine besondere Rolle bei der Anerkennung der Literatur in den anderen Sprachen. Ohne den Übersetzer wäre keine Weltliteratur möglich (vgl. Möckelmann $2004^{1}$ ).

Cornelius Bischoff war ein Übersetzer, der eine wichtige Rolle bei der Anerkennung der türkischen Literatur in Deutschland spielte. Er hatte eine vielseitige Laufbahn, war als Übersetzer, Schriftsteller, Drehbuchautor und Rechtsanwalt tätig. Er interessierte sich für Literatur, Philosophie, Kunst, Film und Musik. Zülfü Livaneli bezeichnete ihn als ein „Prisma“. Je nach Aspekt könne man bei ihm eine tiefe Dimension bzw. eine andere Eigenschaft entdecken (zit. n. Yalçın 2011: 624).

In der vorliegenden Arbeit werden das Leben, die Werke und die Wirkung Bischoffs im Rahmen seiner übersetzerischen Tätigkeit vom Türkischen ins Deutsche besonders hinsichtlich seiner Erfahrungen in der Türkei analysiert. Die Kindheit und

\footnotetext{
${ }^{1}$ Möckelmann, Reiner (Hg.) (2004): YAŞAR KEMAL im Gespräch mit seinem Übersetzer CORNELIUS BISCHOFF und interessiertem Publikum im Deutschen Generalkonsulat am 22. Januar 2004. Broschüre des Generalkonsulats der Bundesrepublik Deutschland in Istanbul. https://tuerkei.diplo.de/blob/1563568/3bc059a3a0a9dde3724584ecd0b70966/datei-yasar-kemal-data.pdf (letzter Zugriff: 29.07.2020).
} 
Jugendzeit Bischoffs, die er als ein „Haymatloser“ in der Türkei verbrachte, werden durch die Angaben, Reportagen, Dokumente und Bilder über sein Leben und seine Stellung als Übersetzer im Werk „Haymatlos Dünya Bizim Vatanımız“ (2011) von Kemal Yalçın und durch eigene Angaben Bischoffs in seinen Artikeln und Reportagen begründet. Die Rolle Bischoffs im Übersetzungsbereich wird im Allgemeinen bezüglich des Zusammenhangs seiner Wurzeln in der Türkei und im Türkischen, der in der Türkei verbrachten Jahre und der Wahrnehmung der türkischen Sprache, Kultur und Gesellschaft betrachtet. Bevor die Bedeutung Bischoffs als ein Übersetzer unternommen wird, werden im folgenden Kapitel kurze Informationen über die deutschtürkischen Literaturübersetzungen vorgestellt, damit die Rolle Bischoffs dabei besser festgestellt werden kann.

\section{Über die deutsch-türkischen Literaturübersetzungen}

Die enge türkisch-deutsche Beziehung wirkt auch auf den Bereich der Übersetzung. Als eine Folge dieser Beziehung hat das Interesse für Literatur und die literarische Übertragung der fremden Kultur und Sprache zugenommen (vgl. Öncü 2019: 158). Wie Öncü festlegt, galt dieses Interesse vor der Gründung der türkischen Republik vielmehr für den militärischen Bereich. Aber nach 1923 vermehrte sich die Anzahl besonders der literarischen Übersetzungen vom Deutschen ins Türkische, oder umgekehrt, um die Kultur des Fremden kennenzulernen (vgl. ebd.: 160). In dieser Hinsicht gewann die Kulturübertragung mehr an Bedeutung. Somit erhielten diejenigen Übersetzer einen Vorteil, die neben beiden Sprachen auch beide Kulturen beherrschten.

Nach Dikici waren die Übersetzungen vom Deutschen ins Türkische oder umgekehrt während der Nazi-Zeit sehr gering (vgl. 2015: 59). Hauptsächlich die damaligen Wissenschaftler und Intellektuellen, die aus Deutschland flohen und in der Türkei Zuflucht fanden, lernten die Türkei und die türkische Kultur näher kennen. Sie versuchten, die politische, wissenschaftliche, soziologische, künstlerische und kulturelle Lage der Türkei in Deutschland vorzustellen. Diese „Haymatloz ${ }^{\text {‘2 }}$ genannten Menschen trugen nämlich sehr wirksam zur Kulturübertragung bei. Sie zeigten ihre Dankbarkeit, indem sie ihre beruflichen Erfahrungen während und nach der Zeit der Gründung der türkischen Republik vermittelten. Die in der Türkei erfassten Erfahrungen hingegen vermittelten sie in Deutschland, als sie zurückkehrten. Sie versuchten im weitesten Sinne, beide Länder zusammenzubringen.

In Deutschland zeigte sich insbesondere in den sechziger Jahren infolge des Zuzugs der türkischen Gasrarbeiter ein klares Interesse an der türkischen Kultur (vgl. Dikici 2015: 63, vgl. Öncü 2019: 161). Viele Verlage versuchten, diese Neugier durch die Übertragung literarischer Werke zu stillen, ,so daß kleine, auf türkische Literatur

\footnotetext{
${ }^{2}$ In Bezug auf die Ortographie muss man hier erklären, dass das Lehnwort „Haymatlos“ auch als „Haymatloz“ geschrieben werden kann. „Zum ersten Mal -in juristischen und politischen Dokumenten, besonders in den Pässen- wurde der Begriff [heimatlos] in die türkische Sprache als „haymatloz“ -mit „Z“- übernommen, aber später wandelte er sich in „haymatlos“ um, vielleicht wegen der einfacheren Artikulation“" (Arslan 2013: 131).
} 
spezialisierte Verlage wie der Ararat-Verlag, der Dagyeli-Verlag und der Unionsverlag entstanden und begannen die Übersetzung türkischer Literatur ins Deutsche zu fördern“ (Bartsch 1999: 8).

Einige Namen treten bei der literarischen Übersetzung vom Türkischen ins Deutsche oder umgekehrt - besonders im Hinblick auf die Kulturübertragung - hervor. Einer davon ist Horst Wilfried Brands. Brands begann damals zum ersten Male, die Werke der Autoren der Nachkriegszeit durch eine „neue“ Methode zu übersetzen: Er dichtete weniger nach, d.h., dass er literarische Werke weniger frei übersetzte. Stattdessen orientierte er sich stärker am Original. Unter den damaligen Übersetzer/Innen war auch Annemarie Schimmel auffällig. Bekannt ist sie durch die Übersetzung sufistischer Texte vom Türkischen ins Deutsche geworden. Ebenso bekannt in der Übersetzungswelt sind der mit sehr guten Deutschkenntnissen Yüksel Pazarkaya und der in Deutschland geborene Zafer Şenocak. Zudem zeigen sich auch die Deutschen mit hervorragenden Türkischkenntnissen: Cornelius Bischoff und Helga Dağyeli-Bohne. Dağyeli-Bohne übersetzte die türkischen Werke überwiegend mit ihrem Mann Yıldırım Dağyeli, dem Gründer des Dagyeli-Verlags (vgl. Bartsch 1999: 8ff).

Bischoff übersetzte 24 Werke vom Deutschen ins Türkische. Nach Angaben der Arbeit Öncüs war er der Dritte unter den Übersetzern, die die meisten literarischen Werke vom Türkischen ins Deutsche übersetzten. Die anderen zwei Namen vor ihm sind Sabine Adatepe und Helga Dağyeli-Bohne. Adatepe und Dağyeli-Bohne studierten Turkologie und waren mit türkischstämmigen Männern verheiratet (vgl. Öncü 2019: 167ff). Dies waren die Gemeinsamkeiten, die sie in der Übersetzungswelt teilten.

Der Zusammenhang Bischoffs mit der Türkei hingegen ist eigentlich vor allem darauf zurückzuführen, dass schon seine Mutter einen engen Zusammenhang mit der Türkei hatte und dass auch Bischoff selbst lange in der Türkei lebte. Er studierte nicht Turkologie, sondern lernte die türkische Sprache und Kultur in der Türkei. Seine Beziehung zur Türkei hielt Bischoff immer für etwas Besonderes anders als die anderen Übersetzer. Er deutete es wie folgt:

Ich hatte da [beim Übersetzen] keine Schwierigkeiten, weil ich ein Jahr lang in Anatolien war. Ich kenne also auch Anatolien gut, nicht nur durch Reisen, sondern dadurch, dass ich ein Jahr dort interniert war und es oft bereist habe. Da habe ich auch die Sprache der Bauern und Dörfler kennen gelernt. Deswegen hatte ich nie so viel Schwierigkeiten wie andere Übersetzer, die als Turkologen nur das offizielle Türkisch gelernt hatten und dann mehr Schwierigkeiten mit dem Übersetzen haben und ihn oft fragen müssen oder ganze Listen mit Begriffen schicken, die sie nicht verstehen. (zit. n. Möckelmann 2004)

Bischoff hatte eine ,profunde Kenntnis der Türkei“ (Möckelmann 2004), was er als ein Literaturübersetzer zu einem Vorteil verwandelte. Wovon er dabei profitierte, wird detailliert im folgenden Kapitel ausgehend von seinem Leben, seiner Wirkung in der Übersetzungswelt und seinen Werken diskutiert. 


\section{Leben, Werke und Wirkung Bischoffs als ein „haymatloser“ Übersetzer in der deutsch-türkischen Literatur}

\section{Leben}

Bischoff ist 1928 als Sohn eines deutschen Zimmermanns und einer lange in Istanbul lebenden Jüdin mit serbischer Abstammung in Hamburg-Harburg geboren. 1939 floh er mit seiner Familie vor dem Nazi-Regime nach Istanbul. Von 1944 bis 1945 war er mit seiner Familie als deutscher Staatsbürger im anatolischen Çorum interniert. 1948 begann er sein Studium an der Juristischen Fakultät in Istanbul und schloss sein Staatsexamen 1954 in Hamburg ab. Er ist ,als 11jähriger Deutscher von 1939 bis zum [...] zwanzigsten Lebensjahr anno 1948 [...] bikulturell in der Türkei aufgewachsen“ (Bischoff 1996: 45). 1961 beendete er sein Praktikum und wurde Rechtsanwalt. Dennoch war er nicht als ein Rechtsanwalt in Deutschland tätig, sondern öffnete nach dem Studium einen Döner-Laden. Trotz seines Jurastudiums bevorzugte er zudem, sich vielmehr mit Kunst, Literatur und Übersetzung zu beschäftigen. Der wichtigste Grund für sein Interesse an der übersetzerischen Tätigkeit war, dass er sein Leben sowohl geistig als auch körperlich hin- und herpendelnd zwischen der Türkei und Deutschland führen wollte, und dass sein Interesse für das Türkische nie aufhörte. Ein anderer Grund war, dass die Beherrschung der türkischen Sprache ihm in Deutschland auch finanziell half (vgl. Yalçın 2011: 440ff). Infolge der zunehmenden Handelsbeziehungen zwischen der Türkei und Deutschland suchten schon 1956 viele Firmen, u.a. „Brechin Co.“, nach jemandem, der die türkische Sprache beherrschen und die Handelskorrespondenz führen konnte. Bischoff begann somit, bei „Brechin Co.“ $\mathrm{zu}$ arbeiten und den Handelskorrespondenzen und Übersetzungen zwischen der Türkei und Deutschland nachzukommen. Dies war seine erste offizielle übersetzerische Tätigkeit. Er übertrug zudem die Lesungen der türkischstämmigen Autoren für das deutsche Publikum (vgl. Möckelmann 2004). Nach dem Tod seines Vaters -zumal das Jurastudium überwiegend der Wunsch seiner Familie war- tendierte er durchaus zur Literatur, Philosophie, Kunst und Übersetzung. In seinen literarischen Übersetzungen profitierte er immer von seinen literarischen, philosophischen und künstlerischen Kenntnissen über die Türkei. Er schrieb Drehbücher und Erzählungen, in denen es meist um türkisch-deutsche Beziehungen ging. 1985 schrieb er den Dokumentarfilm „Hattuşa brennt“ für den NRD. Viele Drehbücher übersetzte er ins Deutsche. Eines davon ist das Drehbuch der Verfilmung „Yer Demir Gök Bakır“ (1987). Nach 1980 konzentrierte er sich vielmehr auf die Filmaufnahme. 1990 drehte er den Film ,Eine Liebe in Istanbul“. In den 90'er Jahren profitierten manche Medienunternehmen, u.a. „Spiegel“, von den Informationen Bischoffs, um die kulturellen, politischen und wirtschaftlichen Nachrichten der Türkei zu erfahren. Er übersetzte somit die türkischen Nachrichten ins Deutsche. Er heiratete Karin. Karin war es bewusst, dass Bischoff eine enge Beziehung zur Türkei hatte, sodass sie ihn oft mit den Türken identifizierte (vgl. Yalçın 2011: 590ff.). Die Synthese der türkisch-deutschen Kultur Bischoffs bildete eine „,beeindruckende Geschichte“ (vgl. Yalçın 2011: 593ff). Daher behandelten viele Filme oder literarische Werke über die türkisch-deutsche Beziehung die Geschichte Bischoffs. Beispielsweise ist „Zuflucht am Bosphorus“ (2001) („Boğaziçi’ne Sı̆̆ındık“) für WDR und TRT einer der 
Dokumentarfilme über das Leben Bischoffs (über das Leben Bischoffs: vgl. Yalçın 2011: 590ff, vgl. Programm Buchmesse RUHR 2010³, vgl. Möckelmann 2004). Oder im literarischen Werk „Haymatlos: Dünya Bizim Vatanımız“ (2011) von Kemal Yalçın geht es um „haymatlose“ Intellektuelle und Wissenschaftler in der Türkei ausgehend von der Geschichte Bischoffs. Nach all seinen Bestrebungen zwischen der Türkei und Deutschland als ein Kulturträger starb Bischoff 2018 in Deutschland.

Der Wendepunkt in Bischoffs Leben war die Zuwanderung mit seiner Familie im Jahre 1939 im Alter von elf Jahren in die Türkei und die Integration in Çorum, was die tiefsten Spuren in seinem Leben hinterließ (vgl. Yalçın 2011: xviii). Auch wenn er nach dem Krieg nach Deutschland zurückkehrte, sagte er sich nie von der Türkei und vom Türkischen los. Denn er hatte immer noch die Verwandten und Freunde sowie die Erinnerungen und Erfahrungen der Kindheit und Jugendzeit in der Türkei. Seine Übersetzungen hielten diesen Zusammenhang im sprachlichen und kulturellen Sinne immer aufrecht. Er sprach „Türkisch so gut wie ein Muttersprachler“. Die türkische Sprache hatte er in Istanbul gelernt. Es war die Standartsprache. Er lernte außerdem den anatolischen Dialekt in Çorum (vgl. ebd.: 3ff). Wie Yaşar Kemal, einer der bedeutendsten Romanciers der türkischen Literatur, folgenderweise betont, wurde Bischoff unter den Türken aufgenommen:

[...] mein Übersetzer Cornelius Bischoff Efendi kennt es ${ }^{4}$, er hat es in Anatolien, in Çorum, wo er auch gelebt hat, gelernt. Und er kann auch das Istanbuler Türkisch, kann also fast so gut Türkisch wie ich. [...]. Und dieser Mann hier kommt aus Çorum in Mittelanatolien (zit. n. Möckelmann 2004).

Alkok betont eine ähnliche Überzeugung über Bischoff wie folgt:

Bei den Übersetzungen aus dem Türkischen bereitet es für Bischoff keine Schwierigkeiten, die Wörter zu verstehen, denn er kennt nicht nur das offizielle Türkisch, sondern auch die Sprache der Bauern und Dörfler, weil er ein Jahr in Anatolien interniert war und Anatolien oft bereist hat (Alkok 2007: 102).

Er ist zwar bekannt als der Übersetzer von Yaşar Kemal, aber sein Ruhm ist eigentlich auch auf die Übersetzung des Dramas „Ali aus Keshan“ von Haldun Taner oder auf das Kinderbuch „Gülibik“ von Çetin Öner zurückzuführen (vgl. Yalçın 2011: 3ff).

Ein weiteres Merkmal, das Bischoff in der Übersetzungswelt auffällig macht, ist der Zusammenhang seiner Wurzeln mit der Türkei. Seine Oma, seine Mutter und sein Vater hatten schon vor ihm einen Kontakt mit der Türkei. Die Ahnen seiner Oma waren unter den aus Spanien geflüchteten und im Osmanischen Reich Zuflucht gefundenen Juden (vgl. Yalçın 2011: 3ff). Sein Vater war ab 1920 für drei Jahre als Zimmermann in Istanbul tätig. Er lernte Bischoffs Mutter in dieser Stadt kennen (vgl. ebd.: 32). Seine Mutter, Berta, war eine in Istanbul lebende Jüdin serbischer Herkunft. Nach der Heirat begannen sie, in Deutschland zu leben. Nachdem die Judenfeindlichkeit aber in Deutschland stieg, flohen sie nach Istanbul mit der Begründung, dass sie die Stadt „Istanbul“" schon kannten und gerne in dieser Stadt leben wollten (vgl. ebd.: 107). In der

\footnotetext{
3 Programm von Buchmesse RUR

file://C:/Users/M\%C3\%BCge/Downloads/Programmbroschuere.pdf (letzter Zugriff: 29.04.2020).

${ }^{4}$ Das anatolische Türkisch
} 
Familie Bischoffs wurde versucht, die Mehrsprachigkeit und den Multikulturalismus aufrecht zu erhalten. Seine Mutter war ohnehin multikulturell und -lingual aufgewachsen. Sie konnte Türkisch, Deutsch und Griechisch (vgl. ebd.: 33ff). Auch sein Vater lernte durch seinen Aufenthalt in Istanbul ein wenig die türkische Sprache (vgl. ebd.: 35ff). Während der Zeit in der Türkei lebte die Familie Bischoff einerseits mit der Hoffnung, nach dem Krieg nach Deutschland zurückzukehren, andererseits versuchten sie, sich in der Türkei zu integrieren, als ob sie immer dableiben würden. In dieser Hinsicht war die Sprache von großem Belang. In der Familie beherrschte nur Bischoff die türkische Sprache am besten, zumal er sie von Tag zu Tag mithilfe seiner türkischen Freunde verbesserte. Denn er war ein extrovertierter Junge, hatte immer Kontakt mit türkischen Freunden und ein großes Interesse an dem Türkischen und der Türkei (vgl. ebd.: 594).

Wie Reiß hervorhebt, ist die literarische Übersetzung ein „wichtiges Medium des gegenseitigen Austauschs unter den Völkern“ (Reiß 1971: 7). Dieser Überzeugung entsprechend wollte Bischoff durch seine Übersetzungen lebenslang den gegenseitigen Austausch unter den Deutschen und Türkischen sicherstellen. Durch seine eigenen Erfahrungen und der Familie in der Türkei hielt Bischoff sich bis zum Tod für eine Brücke zwischen der türkischen und deutschen Kultur (vgl. Bischoff 1996: 44). Eine Brücke zwischen dem Türkischen und Deutschen zu bauen, wollte er nach seinem eigenen Ausdruck, weil er hoffte, „daß sich Diskriminierungen von Türken in Deutschland eindämmen lassen, wenn man diesen Anderen, insbesondere seine Literatur, kennenlernt" (ebd.: 45). Wie er folgenderweise unterstrich, solle weder die eine noch die andere Kultur oder Sprache bei der Identität Bischoffs als Übersetzer überwiegen:

\footnotetext{
Um zwischen Kulturen vermitteln zu können, muß man in mindestens zwei Kulturen heimisch sein. Das trifft in meinem Fall zu, ich bin in der deutschen und der türkischen Kultur aufgewachsen, besser: in diese beiden Kulturen hineingewachsen, und kann mit Goethe behaupten: „Zwei Seelen wohnen, ach, in meiner Brust“, doch im Gegensatz zu ihm kann ich das ,ach“, diesen Ausruf des Bedauerns, streichen, denn ich will weder die eine, noch die andere Seele missen (Bischoff 1996: 44).
}

Nach dem obigen Zitat ist es die Besonderheit Bischoffs, die ihn von den anderen Übersetzern unterscheidet, seine lebenslange Beschäftigung mit der Türkei und dem Türkischen. Sein bikulturelles und bilinguales Leben bildeten eine Einheit. Er war in den beiden Sprachen und Kulturen gleichwertig heimisch, wovon er beim Übersetzen profitierte.

\section{Bischoff als ein ,haymatloser“ Übersetzer und Kulturträger zwischen Deutschland und der Türkei}

Obwohl Bischoff keinen akademischen Hintergrund im Bereich der Übersetzungswissenschaft hatte, war er lange als Übersetzer vom Türkischen ins Deutsche tätig. Nach Yalçın gelang es ihm, „erfolgreiche“ Übersetzungen anzufertigen, weil er ein aufrichtiger Leser war, eine besondere Neigung zur Literatur hatte, und weil 
er sich immer darum bemühte, etwas Neues zu lernen. Er war ein guter Leser; so las er die zu übersetzenden Texte sorgfältig. Er hatte eine besondere Neigung zur Literatur, sodass er selbst Kurzgeschichten oder Drehbücher schrieb. Er versuchte immer, etwas Neues zu lernen. Beispielsweise wurde er nie müde, vor dem Übersetzen die Landschaften und Menschen in einem literarischen Werk zu verfolgen (vgl. Yalçın 2011: 500ff). Er wurde somit als ein Literaturübersetzer zu einem der wichtigsten Kulturträger zwischen der Türkei und Deutschland (vgl. ebd.: xix).

Vermeer setzt bei der literarischen Übersetzung voraus, dass der Übersetzer zuerst ein guter Leser sein soll. Denn er soll vor allem literarisch und ästhetisch verstehen, was er lese und dann soll er das Verstandene literarisch und ästhetisch übertragen: „Der Übersetzer ist ein Leser, der dann versucht, mit den Mitteln seiner Welt, das, was er an Interpretationen interpretiert hat, sprachlich neu zu gestalten“ (Vermeer 2014: 28). Wie oben erwähnt wurde, war Bischoff ohnehin ein guter Leser. Als ein Übersetzer passt er zu dieser Überzeugung Vermeers. Zudem identifiziert Vermeer einen Übersetzer mit einem Regisseur und Schauspieler. Der Übersetzer solle erst dadurch ,in einer anderen Kultur, in einem anderen Raum, zu einer anderen Zeit ,funktionieren“" (vgl. ebd.: 28). Entsprechend dieser Meinung Vermeers „funktionierte“ Bischoff als ein Drehbuchautor und Regisseur in der türkischen Kultur, in der Türkei und zu einer anderen (manchmal früheren) Zeit.

Bischoff wird in dieser Arbeit als ein „haymatloser“ Übersetzer dargestellt. Das Adjektiv „haymatlos“ ist ein Lehnwort in der türkischen Sprache aus dem Deutschen, das im weitesten Sinne diejenigen definiert, die „keine Staatsangehörigkeiten irgendeines Staates“ aufweisen (vgl. TDK 2019 , vgl. Nişanyan $2017^{6}$ ). Im engeren Sinne weist es auf die deutsch-, schweiz-, und österreichstämmigen Intellektuellen, Handwerker, Künstler, Musiker, Politiker und Wissenschaftler hin, die zwischen 19331939 aus Nazi-Deutschland flohen und in der Türkei Zuflucht fanden. Mit dem Stempel „Haymatloz“ wurde ihre Heimatlosigkeit im politischen Sinne niedergeschrieben (vgl. Arslan 2013: 134, vgl. Kemal 2011: xv). Die meisten Deutschstämmigen in der Türkei, die einen großen Beitrag zur deutsch-türkischen Beziehung leisteten, wurden im Jahre 1944 nämlich „Haymatloz“ genannt, weil die diplomatischen Beziehungen zwischen Deutschland und der Türkei nach dem Krieg zum Stillstand kamen und die „Haymatlozen“ gleichwohl ablehnten, zurückzukehren. Die in der Türkei gebliebenen „Haymatlozen“ wurden in Çorum, Kırşehir und Yozgat interniert (Yalçın 2011: 249ff). Bischoff und seine Familie waren unter diesen „Haymatlozen“. Sie wurden mit dem Stempel „Haymatloz“ im Ausweis in Çorum interniert (vgl. ebd.: 255). Mit der Aussage „ein haymatloser Übersetzer“ weist man so zuerst auf die damaligen Lebensjahre Bischoffs in der Türkei hin. Es wird aber zudem gemeint, dass Bischoff sowohl die türkische Sprache und Kultur als auch die deutsche gut beherrschte. Das „HaymatlosSein“ deutet hier nämlich nicht als etwas Negatives auf einen Mangel hin, sondern auf

\footnotetext{
5 Türk Dil Kurumu [TDK] (2019): Genel Türkçe Sözlük. https://sozluk.gov.tr/ (letzter Zugriff: 11.05.2020).

6 Nişanyan, Sevan (2017): Sözlerin Soy Ağacı: Çağdaş Türkçenin Etimolojik Sözlüğü. http://www.nisanyansozluk.com/ (letzter Zugriff: 11.05.2020).
} 
die Pluralität Bischoffs. Wie Müge Arslan in ihrer Arbeit betont, assoziiert der Begriff „Heimatlosigkeit“ vielmehr den anderen Begriff „Weltbürgertum“ durch den Bedeutungswandel infolge der Globalisierung. Denn der „Weltbürgertum-Gedanke schafft jegliche Bindungen und Grenzen ab, und zielt auf das Zusammenleben“ (Arslan 2013: 185). Statt der Zuordnung zur bestimmten Heimat, Nation, Religion, Ideologie oder zu einem bestimmten Staat und Land beschreibt der Begriff „Weltbürgertum“ die „Freiheit, Ungebundenheit, Universalität, Gemeinsinn, Humanität, Menschenfreundlichkeit, Erdensohn, Ganzheit" (vgl. ebd.: 185). In diesem Kontext stellt die Heimatlosigkeit Bischoffs hier die Vielseitigkeit seiner Persönlichkeit und seiner Laufbahn und den Weltbürgertum-Gedanke dar. Das „Haymatlos-Sein“ Bischoffs weist nämlich vor allem geistig auch auf die deutsch-türkische Identität Bischoffs hin.

Bischoff beschrieb sich selbst als denjenigen, der ,in der Türkei gut integriert” war (Bozay 2001: 115). Mit Istanbul hat er eine „besondere“ Verbindung, die durch Erinnerungen und Erfahrungen geprägt war. Schon die erste Liebe erlebte er in Istanbul (vgl. Yalçın 2011: 374). Seine türkischen Freunde nannten Bischoff „Koni““ (vgl. ebd.: 327). Er gewöhnte sich an die türkische Kultur so sehr, dass er sich in Deutschland nach „Simit“, Schwarzem Tee, Fisch, dem türkischen Kaffee, Freunden, Sehenswürdigkeiten und Landschaften der Türkei sehnte (vgl. ebd.: 374ff). Er hatte zwei Seelen, wie oben von ihm zitiert wurde: Die Lebensweise und Gewohnheiten aus der Kindheit waren überwiegend durch Harburg, Deutschland, ausgeprägt. Die Jugendzeit hingegen assoziierte er mit Istanbul und Çorum. Die Ankunft in Deutschland aus der Türkei erweckte daher anfangs ein Fremdheitsgefühl bei ihm (vgl. ebd.: 404). Er war der Meinung, dass ein Leben ohne Istanbul, Beyoğlu, die Türkei keinen Sinn hatte. Deutschland und die Türkei zusammen solle seine Persönlichkeit bilden. Neben Deutschland nehme er auch die Türkei als seine Heimat wahr. Einmal sprach er davon, dass er selbstverständlich die Deutschen gut kenne und ihre Gefühle, Gedanken und Haltungen einfach interpretieren könnte. Nach ihm kannte er auch die Türken und die Menschen in der Türkei ebenso näher und er konnte sie gut interpretieren (vgl. ebd.: 405ff).

Bischoff internalisierte sowohl das Istanbuler Lebensgefühl als auch das anatolische (vgl. Möckelmann 2004). Als er in Çorum ankam, war er sechzehn Jahre alt (vgl. Yalçın 2011: 329). Çorum hatte somit eine wichtige Rolle für ihn beim türkischen Sprach- und Kulturerwerb. Dort fand er die Möglichkeit, „Anatolien kennenzulernen“ und den Dialekt der türkischen Sprache zu erlernen (vgl. Bozay 2001: 115). Somit überwand er sowohl die grammatischen und lexikalischen Grenzen der Sprache als auch deren geistigen bzw. kognitiven Grenzen (vgl. Bischoff 2010: 171).

Im Jahre 2010 war Bischoff Ehrengast der Buchmesse Ruhr, die unter dem Motto „Liestanbul“ organisiert wurde. Das folgende Zitat betont somit nochmal die Besonderheit Bischoffs zwischen der deutschen und türkischen Kultur. Er solle zunächst das Deutsche und Türkische zusammenbringen:

Cornelius Bischoff verkörpert den Inbegriff einer gelungenen Symbiose der zunächst unterschiedlich erscheinenden deutschen und türkischen Kultur und nimmt damit eine authentische Vorbildfunktion für die junge Generation der Deutschtürken ein. Oder um 
es schlicht mit den Worten Yaşar Kemals auf den Punkt zu bringen: „Er ist der deutscheste der Türken und der türkischste der Deutschen“ (Programm von Buchmesse RUHR 2010).

Die türkische Musik und besonders die Volkslieder spielten eine wichtige Rolle für Bischoff. Die türkischen Lieder bezauberten ihn. Als ein Produkt der Traditionen, Bräuche und kulturellen Spuren des Volks vertieften sie die Wahrnehmung Bischoffs auf die türkische Sprache, Kultur bzw. Tradition (vgl. Yalçın 2011: 330ff). Seine ästhetische Auffassung, die er überwiegend durch die Volkskultur verinnerlichte, half ihm beispielsweise beim Übersetzen der Werke Kemals, die vorwiegend mit der Volkskultur bzw. dem Volkslied verschachtelt gestaltet wurden (vgl. Kula 2016: 66).

Bischoff kannte auch die türkische Küche. „Döner“ assoziierte er mit Istanbul und „Pekmez“ mit Çorum. Auch von der türkischen Küche war er tief beeindruckt (vgl. Yalçın 2011: 331). Er öffnete deswegen Döner-Läden auf Bahnhöfen in Deutschland, in denen er fünf Jahre lang Döner und andere türkischen Speisen verkaufte (vgl. ebd.: 455ff).

\section{Wirkung}

Ich hege den Verdacht, dass man eine literarische Übersetzung im allgemeinen für einen mehr technischen Vorgang hält, der erst Aufsehen erregt, wenn sich eine Panne einstellt.

C.Bischoff ${ }^{7}$

Jiří Levý bestimmt drei Phasen der Übersetzbarkeit: das „Erfassen der Vorlage“, die „Interpretation der Vorlage“, die „Umsetzung der Vorlage“ (vgl. 2012: 24). Der Übersetzer soll vor allem den zu übersetzenden Text erfassen. Dieser erste Schritt, der eigentlich bedeutet, ,in den Sinn des Werks“ einzudringen, ist der Grundstein des Übersetzungsprozesses. Dies bedeutet, nicht nur die Sprache zu beherrschen, sondern das Wesen der Sprache tiefgreifend $\mathrm{zu}$ begreifen, weil eine oberflächliche Sprachkompetenz zu Übersetzungsfehlern führen kann. Der Übersetzer muss dabei gleich oder ähnlich klingende Wörter bzw. „die falschen Freunde“ beachten. Zu einem solchen Erfassen muss ein guter Übersetzer zuallererst ein aufrichtiger Leser sein (vgl. ebd.: 25 ff). Auf der anderen Seite fordert seine Übersetzungstheorie das Erfassen „der stilistischen Werte des sprachlichen Ausdrucks, d. h. der Stimmungen, ironischen oder tragischen Untertöne (vgl. ebd.: 27). Dies ist von Belang, weil ohne diese stilistischen Werte die Literarizität bzw. Ästhetizität und eigentlich die Besonderheit des Werks umstritten sind. Außerdem soll der Übersetzer auch das künstlerische Ganze bzw. die „im Werk ausgedrückten Realitäten“, wie die „Gestalten“, das „Milieu der Handlung“ und den ,ideologischen Standpunkt des Autors“ erfassen (ebd.: 28). Damit ist gemeint, dass nicht nur der Text und dessen Sprache oder das Allgemeine im Text erfasst werden

\footnotetext{
${ }^{7}$ zit. n. Möckelmann 2004.
} 
sollen, sondern auch das Einzelne des Textes, sowie die ästhetische und ideologische Tendenz des Autors und die von ihm übertragene Realität. In diesem Kontext ist beispielsweise eine Bewertung gesellschaftlicher Werte, Normen, Traditionen, etc. nach dem Übersetzer unumgänglich. Im Rahmen der Wahrnehmung der dargestellten Wirklichkeit unterscheiden sich ,wörtliche und kreative Übersetzung“ voneinander, wie Levý betont:

\begin{abstract}
Der Hauptunterschied zwischen dem schöpferischen und dem mechanischen Übersetzer besteht darin, daß sich der schöpferische Übersetzer auf dem Wege vom Original zur Übersetzung die Wirklichkeit, von der er schreibt, vorstellt, daß er also über den Text hinaus zu den Gestalten, Situationen und Ideen vordringt, während der unschöpferische Übersetzer den Text nur mechanisch aufnimmt und lediglich Wörter übersetzt. (Levý 2012: 29)
\end{abstract}

In dieser Hinsicht kann der schöpferische Übersetzer durch „Vorstellungskraft und eine durchdachte Interpretation“ die Wirklichkeit im Text rekonstruieren (Levý 2012: 29). Darum profitiert der Übersetzer zuerst von der Kenntnis der kulturellen und gesellschaftlichen Werte der Ausgangssprache. In diesem Rahmen profitierte Bischoff von den kulturellen und gesellschaftlichen Erfahrungen, die er in der Türkei sammelte. Als „preisgekrönter Übersetzer türkischer Literatur“ (Probst 2009 ${ }^{8}$ ) lehnte er die wörtliche Übersetzung ab. Nach ihm konnte die wörtliche Übersetzung auf der lexikalischen und syntaktischen Ebene korrekt sein, aber nicht auf der „Wirklichkeitsebene“, weil die Wirklichkeitsebene der Kulturen sich variiert (vgl. Bartsch 1999: 33ff).

Was Levý in Bezug auf die literarische Übersetzung hervorhebt, ist zudem „die Interpretation der Vorlage“. Dadurch zeigt sich die Kreativität des Übersetzers, besonders wenn von der „Inkongruenz des Sprachmaterials“ die Rede ist. Der Übersetzer, der den Text erfasst, interpretiert ihn, um die vollkommene Bedeutungsübereinstimmung zu schaffen, und muss „die Bedeutung spezifizieren und sich für eine der engeren Bedeutungen entscheiden“ (Levý 2012: 33). Entsprechend zu dieser Annahme Levýs kann festgestellt werden, dass Bischoff die Bedeutung eines Wortes in einer Sprache nicht oberflächlich betrachtete. Wie Yalçın betont, rief die Sprache für Bischoff Erinnerungen und Erfahrungen aus dem Leben wach. Ein Beispiel dafür war der Ausdruck „Buba“ aus der serbischen Sprache, der für Bischoff (und seine Familie) eine besondere Bedeutung hatte (vgl. Yalçın 2011: 371). Es wird oft im Serbischen als ein Liebeswort verwendet. Als ein Liebeswort nannten sein Vater und seine Mutter Bischoff „Buba“. Dies sei aber für sie mehr als ein vierbuchstäbiger Ausdruck, ein ganz besonderer Ausdruck geprägt von den Erinnerungen einer Zuwanderung aus Spanien nach Konstantinopel, aus Konstantiopel nach Serbien, aus Serbien nach Istanbul, aus Istanbul nach Harburg, aus Harburg nach Istanbul. „Buba“ solle eine große Leidenschaft veranschaulichen (vgl. ebd.: 371). Ebenso bedeutete die türkische Sprache etwas ganz Besonderes für die Familie Bischoff: die Sprache eines Volkes, das Bischoff und seine Familie in den schwersten Zeiten umarmte. Sie sei die

\footnotetext{
8 Probst, Maximilian (2009): Exil in der Türkei, Baden im Bosporus. https://taz.de/Exil-in-derTuerkei/!5169120/ (letzter Zugriff: 25.01.2020).
} 
Sprache der Freundschaft und der Treue (vgl. ebd.: 619). Nach Bischoff bekamen beispielsweise einige Wörter im Türkischen durch die Erlebnisse und Erfahrungen einen Sinn. Daher war es nicht so einfach, manche Begriffe wie „Gurbet“ zu übersetzen. Obwohl es mit dem Wort „Fremde“ ins Deutsche übertragen werden konnte, stimmten die Assoziationen der Begriffe „Gurbet“" und „Fremde“ in der deutschen und türkischen Sprache nicht direkt überein. Das ,mittelhochdeutsche [...], vom Heimweh geprägte Wort Elend aus Grimm entspreche mehr dem Wort ,Gurbet““ (vgl. Bischoff 1996: 45). Aufgrund der semantischen Abweichung dieser Begriffe übersetzte Bischoff beispielsweise den Titel des Romans „Gurbet Yavrum“ (1975) (wörtl.: Fremde, meine Kleine) von Aysel Özakın als „Der fliegende Teppich“ (1987). D.h., er übersetzte das Wort „Gurbet" nicht, stattdessen bestimmte er einen neuen Titel ausgehend von dem Hauptthema des Romans. Wenn er gleichwohl das Wort „Gurbet“ als „Fremde“ übersetzte, verfremdete er es wie folgt: ,in der Fremde, im Gurbet, wie die Türken sagen“ (ebd.: 45). Die übersetzerische Tätigkeit fordert daher, solche Besonderheiten (die Singularität) der Sprachen und Kulturen festzustellen, was die Verinnerlichung der Ausgangs- und Zielsprache voraussetzt.

Wie Dağyeli-Bohne feststellt, sollte ein „guter“ Übersetzer vor allem mit der Literatur eng verbunden sein. Um ,äquivalent“ übersetzen zu können, sollte er besser selbst schreiben: „Die besten Übersetzer sind die, welche entweder selbst zur schreibenden Zunft gehören (...) oder mit der Literatur eng verbunden und vertraut sind“" (Dağyeli-Bohne 1989: 53). Auch Reiß setzt schriftstellerische und ästhetische Begabung für eine literarische Übersetzung voraus (vgl. Reiß 1971: 25). Durch so eine Begabung erfasste, interpretierte und reproduzierte Bischoff die Texte der türkischen Literatur nach der Perspektive eines Autors oder eines Lesers. Wie bisher erwäht wurde, schrieb er Drehbücher wie „Gülibik“, „Hattuşa brennt“, „Eine Liebe in Istanbul“" und „Exil Türkei“ (Möckelmann 2004).

Das Kinderbuch „Gülibik“" ${ }^{* 9}$ von Çetin Öner war die erste literarische Übersetzung Bischoffs. Mit diesem Buch fing seine übersetzerische Laufbahn an. Er übersetzte dieses Buch auf Wunsch seines Freundes und half dem Autor bei seiner Veröffentlichung in einem deutschen Verlag. Danach kam die Verfilmung, deren Drehbuch er wieder mit dem Autor zusammen zuerst im Türkischen schrieb und dann ins Deutsche übersetzte (vgl. Yalçın 2011: 490ff).

In den 70er Jahren erzählte der Freund Bischoffs Friedrich Schütte ihm, Besitzer des Hamburger Ernst-Deutsch-Theaters, dass er ein türkisches Theaterstück aufführen wollte. Denn es war nunmehr ein großer Bedarf aufgrund der zunehmenden Anzahl der Türken in Deutschland geworden. Er wartete auf die Empfehlung Bischoffs, weil er ihn für einen der Türken hielt. Nach den Besprechungen mit seinen türkischen Freunden empfahl Bischoff Schütte, das Theaterstück „Keşanlı Ali Destanı“ von Haldun Taner aufzuführen. Mit der Voraussetzung, dass Bischoff es übersetzte, fand Schütte diesen Vorschlag angemessen. Bischoff übersetzte es in einem Jahr. Am 23 April 1981 fand die deutsche Erstaufführung in Hamburg statt. 1985 wurde es in deutscher Sprache mit

\footnotetext{
${ }^{9}$ Die Originalausgabe erschien 1975, Bischoffs Übersetzung erschien 1977.
} 
der Übersetzung Bischoffs als „Die Ballade von Ali aus Keshan“ veröffentlicht (vgl. Yalçın 2011: 504).

Der Schweizer Unionsverlag, einer der wichtigen Verlage Europas der 70er Jahre, fragte 1977 Bischoff, ob er den Roman „Kuşlar da Gitti“ (1978) von Kemal übersetzen wollte. Bischoff hatte zwar bisher keine Romane Kemals gelesen, er kannte aber den Namen Kemals als ein wichtiger Autor der türkischen Literatur (vgl. Yalçın 2011: 504). Der Probetext, den der Verlag ihm zum Übersetzen schickte, gefiel Bischoff. Er entschied sich somit dafür, den Roman zu übersetzen. Er hatte dennoch eine Voraussetzung. Seine Türkischkenntnisse seien nach Bischoff nicht diskutierbar. Wenn der Verlag diesen von ihm übersetzten Text buchstäblich annahm, wie er ihn vorbereitete, wollte er ihn gern übersetzen. Daraufhin akzeptierte der Verlag die Voraussetzung Bischoffs. Das Abenteuer mit den Romanen Kemals begann nämlich durch die Übersetzung „Auch die Vögel sind fort“ (1984) (vgl. ebd.: 505). Dies war ein weiterer Wendepunkt für seine übersetzerische Laufbahn, zumal er nunmehr als der Übersetzer der Werke Yaşar Kemals in der türkischen und deutschen Literatur bekannt wurde. Kemal war ein wichtiger Romancier der türkischen modernen Literatur (vgl. Çiftlikçi 1993: xviii). Nach „Kuşlar da Gitti“ übersetzte er „,Yer Demir Gök Bakır“ (1963) (dt.: Eisenerde, Kupferhimmel-1986), „Yılanı Öldürseler““ (1976) (dt.: Töte die Schlange-1988) und „Ince Memed“ (1955-1987) (dt.: Die Memed-Romane). Die Rezeption der Memed-Romane war in Deutschland weitverbreitet. Mit der deutschen Ausgabe wurden die Memed-Romane damals in Deutschland über 600.000 Mal verkauft (vgl. Yalçın 2011: 505ff und vgl. Möckelmann 2004). Die Popularität Yaşar Kemals in Deutschland wäre von daher ohne Bischoff nicht denkbar (vgl. Möckelmann 2004, vgl. Yalçın 2011: 576). D.h., der größte Anteil des Erfolgs Kemals in Deutschland gehört Bischoff. Der Erfolg Kemals zeigte auch den Erfolg Bischoffs beim Übersetzen. Wie Kemal betont, egal wie erfolgreich ein Roman gestaltet ist, ist er gleichwohl verloren, wenn er nicht von einem ,kompetenten“ Übersetzer übersetzt wird (zit. n. Yalçın 2011: 612). Nach Dilek Dizdar bevorzugen die Autoren deswegen meist auch „unübersetzt in einer Sprache“ zu bleiben, ,als über eine „schlechte“ Übersetzung bekannt zu werden“ (Dizdar 2014: 34). Denn der Autor wünscht sich nicht nur die Wahrnehmung im eigenen Land, sondern auch in den anderen Ländern. Darüber hinaus zielt er bestimmt darauf ab, in der Weltliteratur bekannt zu sein. Daher kommt dem Übersetzer eine wichtige Mission zu. Er ist am Erfolg des Autors beteiligt.

Zafer Şenocak, ein wichtiger Schriftsteller und Übersetzer der türkischdeutschen Literatur kritisiert die oberflächliche Betrachtung der übersetzerischen Tätigkeit. Das Übersetzen der literarischen Werke setze die Wahrnehmung der Tiefenstruktur des Textes voraus. Es solle nicht nur die Übertragung der sprachlichen Elemente, sondern auch der persönlichen Dimension des Autors umfassen (vgl. Şenocak 1989: 60). In diesem Kontext sticht unter den Beziehungen zwischen Bischoff und den Autoren, deren Werke er übersetzte, die Freundschaft zu Kemal besonders hervor. Die Texte, besonders Kemals Texte, übersetzte er nach dem Prinzip der Gegenseitigkeit, d.h. durch den gegenseitigen Gedankenaustausch mit dem Autor (vgl. Möckelmann 2004). 
Die eigentliche Bekanntheit im Bereich der literarischen Übersetzung erlangte Bischoff durch seine Übersetzungen der Romane Kemals, der eine eigenartige Sprache und Schreibweise aufweist und besondere gesellschaftliche, kulturelle, volkstümliche Themen behandelt. Kemal ist bekannt für seine Romane, die sprachlich und inhaltlich das Dorfleben, Anatolien und die Probleme der Bauern thematisieren (vgl. Çiftlikçi 1993: xv). Seine Themen sind nämlich dem Volk immanent. Er überwindet die Grenzen des Dorfs, der Stadt und des Lands durch seine Romane und erreicht das Universale ausgehend vom Regionalen (vgl. Çiftlikçi 1993: 41, vgl. Kula 2016: 64ff). Die langen und komplexen Beschreibungen sind in seinen Werken auffällig (vgl. Bartsch 1999: 15). Die Dialekte, Redewendungen, Sprichwörter, Metapher, die vulgären Ausdrücke, die märchenhaften Erzählungen, die Hinweise auf die türkischen Legenden und Heldenepos usw. sind Elemente seiner Erzählweise, die das Übersetzen erschweren (vgl. Çiftlikçi 1993: 571ff, vgl. Bartsch 1999: 14ff). Seine Schreibweise setzt daher eine mehrdimensionale Sprachkenntnis und Allgemeinbildung über die Türkei voraus. Zum Übersetzen sollen nämlich die Türkei, die türkische Sprache und Kultur, das Volk, die damalige Lebensweise und Gesellschaft, das Anatolien, die Bauern, das Volksleben, und auch Kemal, seine Schreibweise, die Grenzen zwischen dem Gesagten und Gemeinten im Text perzipiert werden. Es ist zudem schwierig, Kemal zu übersetzen, weil seine Schreibweise zwischen dem Traditionellen und Modernen steht (vgl. Bartsch 1999: 15). Er legt einen großen Wert auf mündlich überlieferte Literatur (vgl. Çiftlikçi 1993: 70). Seine Werke zu übersetzen, bedeutet somit, die türkische Volksliteratur in eine andere Sprache zu übertragen. Er hat eine tiefgreifende Beobachtungskraft (vgl. ebd.: 32), die zur gründlichen Darlegung der Einzelheiten dient: Die Farben, Insekten, Blumen, Menschen, bzw. die Natur und die Gesellschaft werden in seinen Werken ausführlich beschrieben, sodass man beispielsweise auch die Blumennamen in Çukurova (vgl. ebd.: 32), die Farbtöne der türkischen Kultur (vgl. Balc1 2015: 231) durch sie lernen kann. In dieser Hinsicht soll der Übersetzer seiner Werke das Allgemeine und Besondere sowohl der Türkei und des Anatoliens als auch Kemals tiefgreifend kennen. Bischoff hatte einen Vorteil darin, dass er neben der türkischen Sprache und Kultur auch Kemal näher kannte. Wegen der engen Beziehung mit Kemal entstand fast keine form- oder inhaltsbezogene Lücke in seinen Übersetzungen der Kemal-Romane (vgl. Yalçın 2011: 624). Über 20 Jahre hinweg arbeiteten sie zusammen. Den Übersetzungsprozess der Romane von Kemal erklärt Bischoff mit einem Beispiel wie folgt:

\footnotetext{
Neulich habe ich ihn [Kemal] angerufen, nachdem ich alles durchgesucht hatte und ihn gefragt, was „abeş” heißt. Es ging um eine Pferdefarbe. Ich hatte viele türkische Wörterbücher durchgeschaut und nichts gefunden. Weil er die anderen Pferdefarben schon erwähnt hatte, dachte ich, es könnte ein Falbe sein, also eine helle Farbe. Falbe konnte ich ihm auch nicht übersetzen, so habe ich ihn gefragt, ob die Farbe des Pferdes ins Gelbe ginge. Bis ich dann dahinter gekommen bin, dass es evtl. auch aus dem Beigen kommen kann. Er sagte dann, dass es ein ins Gelbe gehendes Pferd, also ein Falbe ist". (zit. n. Möckelmann 2004)
}

Nach Bischoff sollte sich ein Übersetzer mit dem Autor identifizieren. Besser gesagt solle er ,,in der Haut des Autoren“ verschwinden, „den er übersetzt“ (Bischoff 1989: 
35). Die Haut des Autors meine die Ausgangssprache und -kultur, d.h., die Türkei, die türkische Sprache, und alles über den Autor. In dieser Richtung entwickelte sich mit der Zeit eine enge Freundschaft zwischen Bischoff und Kemal. Diese Beziehung glich einer Bruderschaft. Sie war mehr als nur eine Beziehung zwischen dem Autor und Übersetzer (vgl. Yalçın 2011: 506). Er verinnerlichte Kemal so tief, dass ein großes Bild Kemals in seinem Haus an der Wand hing. Den Grund dafür erklärte Bischoff mit der Identifikation zwischen Autor und Übersetzer (vgl. Yalçın 2011: 505ff). Denn ,je unvollkommener die Identifikation mit dem Autor, desto sichtbarer rückt der Übersetzer in den Vordergrund, desto mehr verliert seine Arbeit an Güte" (Bischoff 1989: 35).

Vor dem Übersetzen machte Bischoff Forschungen über die Tiefenstruktur des Textes; über den Ort, über das Volk, über den Dialekt, über die Figuren. Er bereiste beispielsweise Çukurova und das Taurusgebirge, blieb da Jahre lang, um die Dörfer, Berge, Täler und Menschen zu besichtigen, die Kemal in seinen Romanen beschreibt (vgl. Yalçın 2011: 624). „Er lebt in ständigem Dialog mit Yaşar Kemal und hat sich durch viele Reisen mit allen Orten der Helden Yaşar Kemals vertraut gemacht" (Möckelmann 2004).

Kemal nahm Bischoff als denjenigen an, der ihn in Deutschland reproduzierte, und der sowohl den türkischen als auch den deutschen Literaturkreis bereicherte (vgl. Yalçın 2011: 604, vgl. Möckelmann 2004). Ebenso nach Livaneli gab Bischoff Kemal in dem deutschen Literatur- und Kulturkreis eine neue Seele, sodass man seine Übersetzungen nicht nur als eine bloße wörtliche Übersetzung, sondern auch als ein Reproduzieren betrachten sollte (vgl. Yalçın 2011: 624).

Nach Helga Dağyeli-Bohne muss der literarische Übersetzer das Politische, Soziale, Kulturelle der Ziel- und Ausgangssprache gut kennen (Dağyeli-Bohne 1989: 44), zumal sie den wesentlichen Hintergrund der Handlung im Text bilden. Zudem kommen auch das Wirtschaftliche, Philosophische, sogar manchmal das Medizinische. Eigentlich unterscheiden sich dabei „das Übersetzen“ und „das Übertragen“. Im Rahmen des Übertragens kann jedes Wort „wörtlich“ von einer Sprache in die andere transferiert werden (vgl. Dağyeli-Bohne 1989: 45). Das Übersetzen aber sei mehr als „eine wörtliche Übertragung“, was das Kulturelle, Politische, Soziale, Alltägliche und das Besondere, sogar das Religiöse usw. beherbergt (vgl. ebd.: 45ff). Die folgende Meinung von Dağyeli-Bohne zeigt eigentlich, wie „effektiv“ die Übersetzungen Bischoffs sind. Vor allem sei er ein Übersetzer, der die Nuance zwischen dem Übertragen und Übersetzen bemerkte. Mit so einem Bewusstsein zum Unterschied zwischen dem Übersetzen und Übertragen übersetzte er die literarischen Texte, besonders von Kemal, der eine antiquierte Sprache hatte (vgl. ebd.: 48). Ansonsten hätte er Kemal ohne die Beherrschung des Kulturellen, Politischen, Sozialen, Alltäglichen nicht gründlich übersetzen können: „Wenn man [...] der zentralasiatischen Kultur und Religion unkundig ist, dann kann man die meisten Romane Kemals vielleicht ins Deutsche übertragen aber nicht übersetzen“ (ebd.: 50). In diesem Kontext war es zwar eine schwierige Sache, Kemal zu übersetzen, aber gleichwohl für Bischoff eine gute Möglichkeit, seinen eigenen Erfolg und seine Übersetzerschaft zu zeigen. 
Die Romane Kemals, die er ins Deutsche übersetzte, sind wie folgt (vgl. Unionsverlag 2020 ${ }^{10}$ ): Kuşlar da Gitti - Auch die Vögel sind fort (1984), Ölmez Otu Das Unsterblichkeitskraut (1986)/(Die Anatolische Trilogie III), Yer Demir Gök Bakır Eisenerde, Kupferhimmel (1986)/ (Anatolische Trilogie II), Yılanı Öldürseler - Töte die Schlange (1988), İnce Memed III - Das Reich der vierzig Augen (1993), Deniz Küstü - Zorn des Meeres (1996), Kimsecik I - Salman (1999), Firat Suyu Kan Akıyor Baksana - Die Ameiseninsel (2001) (Die Inselromane I), Höyükteki Nar Ağacı - Der Granatapfelbaum (2002), Ince Memed IV - Der letzte Flug des Falken (2003) (Memedromane IV), Karıncanın Su İçtiği - Der Sturm der Gazellen (2006) (Die Inselromane II), Tanyeri Horozları - Die Hähne des Morgenrots (2008) (Die Inselromane III).

Die Werke der anderen türkischen Autoren, die Bischoff übersetzte, sind wie folgt (vgl. Tonkul 2018 ${ }^{11}$ ): „Bitte nix Polizei“ von Aras Ören (1981), „Lachend sterben“ von Haldun Taner (1985), „Der fliegende Teppich“ von Aysel Özakın (1987), „Holzpantinen“ von Necati Cumalı (1993), „Aus dem Leben verbannt“ von Aras Ören (1994), „Ein Frühstück in Kaş“ von Aras Ören (1999), „Der Blick aus meşnem Fenster“ von Orhan Pamuk (2006), „Sieht jemand den blauen Vogel?“" von Çetin Öner (2010), „Der letzte Tscherkesse“ von Çetin Öner (2004), „Das Waisenküken“ von Rıfat Ilgaz (2010), „Kopfstand“ von Aras Ören (2014).

Die Drehbücher und Dokumentarfilme von/über Bischoff sind wie folgt: Eine Liebe in Istanbul / İstanbul'da Bir Aşk (1996), Hatutaş Yanıyor / Hattusa brennt (1985), Zuflucht am Bosporus / Boğaza Sığınanlar (2001) und Exil Türkei / Sürgün Türkiye (1991), Import - Export. Eine Reise in die deutsch-türkische Vergangenheit / ImportExport - Alman-Türk geçmişine bir yolculuk (2006).

Die Auszeichnungen Bischoffs sind wie folgt: Gülibik (1978) - Ehrenpreis der Universität Padua, Ehrenpreis des türkischen Kulturministeriums (1995), Ehrenbürgerschaft von Çorum (2011), der deutsch-türkische Übersetzerpreis Tarabya (2012).

Die Tätigkeiten Bischoffs zwischen Deutschland und der Türkei als Kulturträger waren nicht nur mit seinen Übersetzungen begrenzt. Er unterstützte viele seiner türkischen Freunde in Deutschland. Dank ihm konnten seine türkischen Freunde (besonders die sich mit der Kunst beschäftigten) Kontakte in Deutschland aufnehmen. Seine Brückenfunktion war auch dabei unbestreitbar. Neben Haldun Taner, Zülfü Livaneli und Yaşar Kemal half er auch dem Maler Orhan Peker dabei, sich in Deutschland vorzustellen. Bischoff organisierte einige Ausstellungen Pekers in Deutschland (vgl. Yalçın 2011: 594). Auf diese Weise wäre der Kulturkontakt zwischen Deutschland und der Türkei ohne Cornelius Bischoff unvollständig (vgl. ebd: 594).

\footnotetext{
10 Unionsverlag (2020): Übersetzungen von Cornelius Bischoff. http://www.unionsverlag.com/info/person.asp?pers_id=1320 (letzter Zugriff: 21.08.2020).

11 Tonkul, Hatice (2018): Biogramm von Cornelius Bischoff. https://translex.ege.edu.tr/tr6236/cornelius_bischoff.html (letzter Zugriff: 03.05.2020).
} 
Zusammenfassend ist die Übersetzung, in einem weitesten Sinne immer Kulturarbeit, in einem engeren Sinne Spracharbeit: Arbeit mit der anderen und der eigenen Kultur, Arbeit mit und an der eigenen Sprache“, wie Werner Koller betont (Koller 2011: 54). Beide haben ein dialektisches Verhältnis. Erst somit kann man den tiefen Zusammenhang zwischen dem Gesagten und Gemeinten im Text begreifen oder

Redewendungen, Sprichwörtern, Legenden, Liedern, Ausrufewörtern und Anredeformen einen Sinn verleihen und den Text übersetzen. Das Übersetzen erfordert nämlich einen Kultur- und Sprachkontakt zwischen Ausgangs- und Zielsprache und kultur. Bischoff hatte die Möglichkeit, die Sprache und Kultur in der Türkei kennenzulernen. Anhand seiner sprachlichen und kulturellen Erfahrungen in der Türkei bezweckte er lebenslang, dem deutschen Leser sowohl die türkische Sprache als auch die türkische Kultur vorzustellen (vgl. Bischoff 1996: 44). Bei ihm waren somit die Grenzen zwischen dem Deutschen und Türkischen sowie zwischen der Türkei und Deutschland aufgehoben. Er pendelte zwischen Istanbul, Hamburg, Çorum, Essen sowie zwischen dem Türkischen und dem Deutschen hin- und her. Es wurde für ihn aber nie zu einem negativen Dazwischenstehen, sondern nahm Bischoff sein Dazwischenstehen als eine Bereicherung, als eine Vielfalt wahr (vgl. Yalçın 2011: 625).

\section{Die Übersetzungstechnik Bischoffs und einige Beispiele aus seinen Übersetzungen}

Die vorliegende Arbeit soll keine Übersetzungsanalyse darstellen, in der die Übersetzungen Bischoffs diskutiert werden. Sie zielt vielmehr darauf, Bischoff als Kulturträger, seine Übersetzerschaft und die Wirkung seiner Übersetzungen zwischen der deutschen und türkischen Literatur im Rahmen der Jahre, die er in der Türkei als ein „haymatloser" Jugendliche verbrachte, zu bewerten. In diesem Kapitel werden daher einige hervorstehende Beispiele seiner Übersetzungen im Zusammenhang mit seinem theoretischen Standpunkt zur literarischen Übersetzung aufgeführt.

Die Übersetzungsart Bischoffs in Bezug auf die kulturbedingten Elemente ist dem Text entsprechend manchmal ,adaptierend“, manchmal „transferierend“. Nach Kollers Übersetzungstheorie kulturbedingter Texte ersetzen ,die AS-Textelemente, die spezifisch in der AS-Kultur verankert sind“ bei der adaptierenden Übersetzung durch Elemente der ZS-Kultur. Bei der „transferierenden Übersetzung“ hingegen werden ,die kulturspezifischen AS-Elemente als solche im ZS-Text" vermittelt (Koller 2011: 55). Sprachbedingt unterscheidet Koller „die sich einpassende Übersetzung“ und „die verfremdende Übersetzung“. Bei der ,sich einpassenden Übersetzung“ (,auf das Deutsche bezogen: verdeutschende Übersetzung") werden die sprachlich-stilistischen Elemente im AS-Text nach den Normen des ZS-Textes vermittelt. Bei der „verfremdenden Übersetzung“ bleiben die sprachlich-stilistischen Normen des ASTextes im ZS-Text aufbewahrt (Koller 2011: 55). Bischoffs Übersetzungen sprachbedingter Elemente sind der Satzstruktur oder Bedeutungsstruktur entsprechend manchmal „die sich einpassende Übersetzung“ machmal „,verfremdende Übersetzung“, wie es im Folgenden mit einigen Beispielen zu begründen versucht wird. Der Grund dafür, wie oben erwähnt wurde, war das Ziel Bischoffs beim Übersetzen. Bischoff zielte 
darauf ab, dass der deutsche Leser die türkische Sprache und Kultur kennenlernte und auch der deutsche Leser beim Lesen türkischer Literatur einen hohen ästhetischen Genuss in Bezug auf die Sprache und Kultur empfinden konnte (vgl. Möckelmann 2004). Er glaubte daran, dass er dies am besten durch die Kinder- bzw. Jugendbücher schaffen konnte (vgl. Bischoff 1996: 45). Mit einer solchen Überzeugung übersetzte er zuerst ein Kinderbuch: „Gülibik“. Es ist die Geschichte der Freundschaft zwischen einem armen Kind aus Anatolien und einem lebendigen Hahn. Es ist die Erzählung der kindlichen Naivität, des Anatoliens und des Dorfslebens voll mit Dialekten und regionalen Ausdrücken. Bischoff übersetzte schon den Titel des Buchs mit der Einfügung einer Erläuterung als „Gülibik der Hahn“, indem er betonte, dass Gülibik ein „lebendiger“ Hahn, bzw. kein Spielzeug, ist und warum das Kind ihn „Gülibik“ nennt:

Onun çocukluğu boyunca bir tek oyuncağı oldu: bir horoz, gülibikli bir horoz! (Öner ${ }^{12}$ 1993: 3).

Aber einmal während seiner ganzen Kindheit bekam er doch ein Spielzeug: Einen Hahn! Einen lebendigen Hahn mit einem purpurroten Kamm! ${ }^{13}$ (Öner 1993: 3)

„Gülibik“ ist der Eigenname des Hahns, den das Kind erfindet. Bischoff erklärte ihn schon am Anfang mit den eingefügten Sätzen. Wie folgt betonte Bischoff die eventuelle Entsprechung des Worts „Gülibik“ im Deutschen, damit es eine Vorstellung auch bei dem deutschen Leser erwecken kann. Ansonsten könnte der türkische Eigenname im Deutschen nichts Besonderes assoziieren. Das Besondere im Wort ist die Zusammenfügung der Wörter im Türkischen „Gül“ und „İbik“, was im Deutschen dem Wort „Rosenkamm“ entspricht. Im Deutschen pointierte Bischoff durch die folgende Einfügung darauf, dass das deutsche Wort „Rosenkamm“ als die Zusammensetzung der Wörter „Rosen“ und „Kamm“ das türkische Wort „Gülibik“ besser darstellen konnte:

Gülibik, o yoksul çocuk için oyuncak olmaktan öte birşeydi: Bir dost, bir arkadaş, bir umuttu (Öner 1993: 3).

Gülibik, so nannte der Junge den Hahn, was in seiner Sprache so viel wie Rotkamm oder besser Rosenkamm bedeutet. Gülibik war aber mehr als nur ein Spielzeug. Er war für den Jungen ein Freund, ein Gefährte und eine Hoffnung (Öner 1993: 3).

Im Vorwort von „Gülibik“ steht eine weitere Erläuterung Bischoffs, die betont, dass der Protagonist ein Kind aus Anatolien ist. Hier pointierte Bischoff durch seine Kenntnis über den kulturellen, geographischen und ökonomischen Hintergrund der Türkei darauf, dass sich die Konjunkturlage und das kulturelle Leben in der Türkei je nach Region unterscheiden. Das arme Kind in „Gülibik“ gehört zu dem anatolischen Gebiet. Es hat ein armes Leben, aber besser ein naives. Die menschlichen Eigenschaften wie Toleranz, Freundschaft sowie Liebe überwiegen in seinem Leben, nicht das Geld:

Tahtadan atları, kurşundan askerleri, lastikten topları, plastikten arabaları, kısaca, parayla satın alınabilen hiçbir oyuncağı olmamış, yoksul bir çocuğun öyküsüdür bu öykü. (Öner 1993: 3)

\footnotetext{
${ }^{12}$ Das Kinderbuch „Gülibik der Hahn“ wurde 1993 zweisprachig von Verlag an der Ruhr veröffentlicht. Auf der einen Seite steht links der türkische Originaltext und rechts die deutsche Übersetzung.

${ }^{13}$ Hervorhebungen im Text sind von der Verfasserin.
} 
Dieses ist die Geschichte eines Jungen aus Anatolien. Weil er arm war, bekam er niemals Spielzeug. Weder Pferde aus Holz noch Soldaten aus Zinn, weder Gummibälle noch Plastikautos, denn diese Dinge konnte man nur kaufen, wenn man Geld hatte (Öner 1993: 3).

Wie in der obigen Übersetzung bemerkt wird, ist die Interpretation der Texte für die Zielrezipienten beim literarischen Übersetzen von großem Belang. Der wichtigste Punkt beim Übersetzungsprozess ist ohnehin für Levý die „Umsetzung der Vorlage“. Dabei verlangt man vom Übersetzer ,eine künstlerisch gültige Umformulierung der Vorlage“ (Levý 2012: 46). Durch das Reproduzieren des Interpretierten zeigt der Übersetzer seine Kreativität, Vorstellungskraft, Disziplin und ästhetische Rezeption. Neben dem Autor ist er nun an dem Werk beteiligt. Levý nimmt so an, dass der Erfolg des Autors ausschließlich mit seinem Werk zu tun hat. Der Erfolg des Übersetzers hingegen verwandle sich zu einem Doppelerfolg durch die Betrachtung und Übertragung des Werks. Der Übersetzer könne es durch, „die Vorstellungsgabe, die Fähigkeit zu objektivieren und die stilistische Begabung“ schaffen (Levý 2012: 59ff). Alkok versucht, die ,ästhetische und kreative Übersetzungsart" Bischoffs durch die Übersetzung der folgenden Redewendung $\mathrm{zu}$ begründen. Bischoff übersetzte die Redewendung „sinek kaydı tıraş“ (wörtl.: fliegenrutschende Rasur) in „Ameiseninsel“ (2001) Kemals mit einer Wortneuschöpfung als „fliegenrutschenglatt“ (Alkok 2007: 176ff). Entsprechend zu Levýs Meinung setzte er die Vorlage um, indem er eine künstlerisch gültige Umformulierung durch eine Wortneuschöpfung erreichte. Darüberhinaus verfremdete Bischoff den Ausdruck mit der Zugabe, dass Türken es genauso zu sagen pflegen. Dem deutschen Leser wird dadurch klar, dass diese Wortneuschöpfung eine wörtliche Übertragung aus der türkischen Kultur ist:

Usturasını uzun uzun kılavladı, sinek kaydı bir tıraş oldu. (Kemal 2004: 140)

Er seifte sich ein, zog sorgfältig die Klinge ab und schabte die stoppelige Haut fliegenrutschenglatt, wie die Türken sagen (Kemal 2001: 165).

Beim Übersetzen der Romane Kemals hat die Kenntnis der Farben bzw. der türkischen kulturellen und traditionellen Farbtöne eine große Rolle. Auch dabei zeigt sich die Kreativität Bischoffs. In diesem Rahmen analysiert Tahir Balc1 die Übersetzung der Farben im Roman „Auch die Vögel sind fort“. Die Farbausdrücke unterscheiden sich je nach der Kultur von Land zu Land. Auf diese Weise gewinnt die Übersetzung der Farbausdrücke als ein Merkmal der kulturellen und sprachlichen Besonderheit an Bedeutung. Um dies zu betonen, stellt Balc1 43 türkische Begriffe für die Bezeichnung unterschiedlicher Farbtöne beim Roman Kemals fest, die bei der Übersetzung 50 deutschen Wörtern entsprechen. Diesem ist zu entnehmen, dass das Fehlen der direkten Entsprechung der Farbwörter vom Türkischen ins Deutsche Bischoff dazu bewegten, Erläuterungen einzufügen, was die Anzahl der Farbausdrücke in der deutschen Ausgabe vermehrte (vgl. Balc1 2015: 231). Er übersetzte die türkischen Farbtöne wie „duru mavi“ (duru: rein, mavi: blau) als „dunkelblau“, „som mavi“ (som: rein, echt, mavi: blau) als „reinstes Blau“ oder „tiefblau“ und ,yeşilimtırak“ als „,grünlich“ (vgl. ebd.: 226). Das Farbwort in „Gülibik“ „pembecik“ (pembe: rosa, -cik: Verkleinerungssilbe: chen) kommt als ,rosasot“" in der deutschen Ausgabe vor (Öner 1993: 6). Der Ausdruck 
„şimşek mavisi“ (blitzblau) (Kemal 1999: 7) wird als „bläulich glitzernd“ (Kemal 1986: 5) übertragen. Bischoff versuchte nämlich, für die Farbwörter im Türkischen meistens eine adaptierte Entsprechung im Deutschen zu finden.

Wie es sich im folgenden Beispiel aus dem Werk „Unsterblichkeitskraut“ (1986) Kemals zeigt, sind die Werke Kemals reich an den detaillierten Beschreibungen von Landschaften des Taurusgebirges und Çukurovas sowie Personifikationen und Analogien. Wie bisher erwähnt wurde, bereiste Bischoff diese Landschaften, blieb ein Jahr lang in Çukurova, um die Dörfer, Berge, Täler selbst zu entdecken, bevor er sie für die deutschen Leser übersetzte. Er versuchte, die Beschreibungen der deutschen Sprache zu adaptieren, damit der deutsche Leser sie einfach verstehen kann. Für jede Beschreibung versuchte er dadurch, eine äquivalente deutsche Entsprechung zu finden. Beispielsweise übersetzte er den mundartlichen Ausdruck „koyak“ als „Schlucht“, oder den Ausdruck ,ak bir karanlık“ als „fahles Licht“ statt wörtlich als ,eine weiße Dunkelheit". Denn dem Kontext entsprechend ist hier eigentlich auch im Türkischen „das Licht“ oder „das Glänzen“ hervorzuheben:

\begin{abstract}
Silme bir ay ışı̆̆ı vardı. A ğaçlar, otlar, tümsekler, tepeler bu gümüş pırıltsında sallanır, genişler, uzar gibiydi. Dereleri, koyakları ak bir karanlık doldurmuş, Anavarza kayalığının gölgesini güneye Ceyhan ırmağını uçsuz bucaksız ovanın üstünde eritilmiş gümüş ışıltısında durgun, sessiz, kımıltısız kayalıkların gölgesinde bir süre yitiyor, kayalıkları geçince de parlak kıvrıltısını yeniden sürdürüp akıyordu (Kemal 1999: 7)

Die Nacht war mondhell. In silbernem Glanz schienen Bäume, Gräser, Bodenwellen und Hügel zu schwanken, sich zu dehnen, länger zu werden. Fahles Licht füllte Bäche und Schluchten, warf die Schatten des Anavarza-Felsens auf die Wasser des Ceyhan, die silbern, träge und still durch die endlos schimmernde Ebene dahinflossen, lautlos in die Schatten der Felsen tauchten und nach einer Weile hinter ihnen wieder in glänzenden Windungen dahinströmen. (Kemal 1986: 5)
\end{abstract}

Die übliche Übersetzungsmethode Bischoffs ist die adaptierende, interpretative, kreative und freie Übersetzung (vgl. Bartsch 1999: 122, 128). Er fügte meist Übergangs- oder Füllwörter in die Erzählung ein, „die das jeweilige Geschehen entweder kommentieren oder zu einer stärkeren Veranschaulichung beitragen“ (vgl. ebd.: 122), wie es oben kurz betont wurde. Dabei fallen auch die Titel der Werke auf, die Bischoff meist frei übersetzte. Beispielsweise übersetzte er den Titel „Ince Memed III“ Kemals nicht als „Ince Memed III“, sondern als „Das Reich der Vierzig Augen“. Dies zeigt „das zentrale Thema des Romans“, was den Hauptraum der Handlung beschreibt (vgl. ebd.: 103). Ein weiteres Beispiel dafür ist der Titel „Frrat Suyu Kan Akıyor Baksana“ (1998) von Kemal, den er als „Ameiseninsel“" übersetzte. Ausgehend von dem Hauptort im Werk bestimmte er den deutschen Titel als „Ameiseninsel“, weil die Ameiseninsel eine wichtige Rolle für die Haupthandlung spielte.

Sprachbedingt bevorzugte er manchmal auch die verfremdende Übersetzungsart. Besonders die kulturellen, religiösen und traditionellen Ausdrücke bzw. die Redewendungen und Sprichwörter oder die Ausdrücke des dialektbedingten Sprachgebrauchs übersetzte er zwar überwiegend wörtlich, aber er fügte meist Übergangs- oder Füllwörter in die Erzählung ein, wie bereits oben betont wurde. Das Sprichwort „İnsanoğlu çiğ süt emmiştir“ in „Das Reich der Vierzig Augen“ übersetzte 
Bischoff beispielsweise als „Die Menschenkinder sind undankbar, sie haben rohe Milch gesaugt“ (Bartsch 1999: 114). Die Redewendung „Eli ayağı tutmaz oldu“ (Kemal 1999: 8) in „Ölmez Otu“ adaptierte er als „Arme und Beine versagten ihm den Dienst“ für den deutschen Leser (Kemal 1986: 6). Bischoff selbst erklärte seine Übersetzungsart der Redewendungen wie folgt:

\begin{abstract}
Ich versuche, sie möglichst im Original wiederzugeben. Ich muss das natürlich so übersetzen, dass der deutsche Leser merkt, dass es nicht von Yaşar Kemal stammt, sonst dürfte ich das gar nicht machen, weil der deutsche Leser dann denken könnte, es sei ein wunderschöne Metapher Yaşar Kemals, dabei ist die Redewendung schon 500 Jahre alt. Ich muss diese Redewendung dann definieren, und der deutsche Leser stellt fest, dass sie nicht von Yaşar Kemal kommt. Ich kann z.B. nicht wörtlich übersetzen "su gibi git, su gibi gel", also "geh wie Wasser, komm wie Wasser!" Ich habe lange überlegt, wie ich es übersetzen soll, als die Leute einen Eimer Wasser einem Scheidenden hinterher schütteten. Denn bei uns weiß wahrscheinlich nur noch der Dachdecker, dass Wasser immer seinen Weg findet. Ich habe dann "geh wie Wasser, komm wie Wasser!", in "Geh so leicht wie das Wasser fließt ...", und der deutsche Leser erkennt an der poetischen Wendung, dass es sich um eine Redewendung handelt. Ja, Sprichwörter sind unter diesem Gesichtspunkt schwer zu übersetzen, und ich muss mir von Fall zu Fall immer etwas überlegen“. (zit. n. Möckelmann 2004)
\end{abstract}

Diese Aussage Bischoffs trifft auf das äquivalente Übersetzen zu, bei der es um eine Deckungsgleichheit in zwei Sprachen geht. Nach ihm beschränkt sich das äquivalente Übersetzung ,nicht nur auf die Auswahl deckungsgleicher Begriffe, oder die im Satzzusammenhang den Sinn der Aussage ergeben, sondern es trifft, [...] auch auf die Formen der Grammatik zu, die in einer Sprache aussagekräftiger sind als in einer anderen“" (Bischoff 1989: 39). In diesem Kontext übersah Bischoff auch die Formen der Grammatik im Text nicht. Beispielsweise übernahm er die meisten türkischen Begriffe und Namen in „Das Reich der Vierzig Augen“" nach der türkischen Schreibweise, mit den türkischen Buchstaben wie , ,̆ ç, c“, wie es bei den Wörtern „Yobazoğlu“ und "Çiçeklideresi“ der Fall ist. Zudem findet man darin „einige wenige Beispiele“, in denen er sie gemäß der Aussprache ins Deutsche niederschreibt:, wie ,inşallah“ „inschallah“, „cilpirti“ - „Dschilpirti“, „maşallah“, -,Maschallah“ oder „Padişah“ „Padischah“ (Bartsch 1999: 103ff).

Die sich einpassenden Ausdrücke nach der Transkription sieht man auch wie folgt in „Gülibik“:

Civcivlerse, ilk kez bu kadar gür, bu kadar çirkin bir ses duyduklarından olacak korkmuşlar, gagacıklarını havaya dikip bağırmaya başlamışlardı: „Cik! Cik! Cik! (Öner 1993: 8)

Die Küken, wohl erschreckt von der mächtigen und häßlichen Stimme des Esels, streckten ihre Schnänel in die Höhe und piepsten: „Tschik! Tschik! Tschik! (Öner 1993: 8)

Die Beinamen übertrug Bischoff meist „als appositionelle Beifügung“. Beispiele dafür in „Das Reich der Vierzig Augen“ sind wie folgt: „Kara İbrahim“ - „Ibrahim der Schwarze“, „Köstebek Bekir“ - „Bekir der Maulwurf“, „Tazi Tahsin“ - „Tahsin der Windhund“" (Bartsch 1999: 105). 
Auch beim Übersetzen der kulturbedingten Ausdrücke fügte Bischoff meist Kommentare ein. Er weichte nicht vom Werk ab. Sein Ziel bei der Übersetzung kulturbedingter Elemente war es, die Struktur aufrechtzuerhalten.Türkische Begriffe und kulturelle Spezifika wurden dementsprechend von Bischoff nicht als Fremdwörter unübersetzt übernommen, sondern entweder durch ähnliche Entsprechungen im Deutschen oder durch eine erklärende Beschreibung des jeweiligen Gegenstandes wiedergegeben (Bartsch 1999: 106). Ein Beispiel dafür sind die Ausdrücke in „Das Reich der Vierzig Augen“ wie „ekşi ayran“- „saueren Joghurttrank“, „çarik“ „Opanke“ oder „,dürüm yufka ekmek“ -,,gerolltes Fladenbrot““ (Bartsch 1999: 106).

Die profunden Türkischkenntisse Bischoffs reichten bis zum Wissen über die alte Lebensweise der Türken. In „Gülibik“ spricht man beispielsweise von „düven sürmek“, d.h.; „Ochsen treiben“. In den vergangenen Jahren in Anatolien war es dem Bauern üblich, die Ochsen zu treiben. Um dies auch für den deutschen Leser verständlich zu machen, erklärte Bischoff im Deutschen, wie man die Ochsen treibt:

\footnotetext{
Yemekten sonra Babam: „Sen biraz düveni sür, ben de şuracıkta kestireyim“ deyip gölgeliğe uzandı. Yüzüne de mendil ıslatıp örttü (Öner 1993: 42).

Nach dem Essen sagte mein Vater: „Treib du die Ochsen jetzt ein bißchen. Ich werde hier ein Schläfchen machen“. Dann legte er sich in den Schatten und deckte sein Gesicht mit einem nassen Taschentuch zu. Die Ochsen waren vor ein großes Brett gespannt, das auf der Unterseite aufgerauht war. Auf dem Brett war ein kleiner Hocker. Ich setzte mich darauf und trieb die Ochsen in großen Kreisen über das ausgebreitete Getreide. Dabei lösten sich die Weizenkörner aus den Ähren (Öner 1993: 42).
}

Bischoff achtete auf die ,falschen Freunde“, die die Übertragung zwischen den Sprachen erschweren und Interferenzfehler verursachen. Alkok begründet dies mit einem Beispiel aus dem Werk „Ameiseninsel“. Den Ausdruck „Kayığa göz kulak olun!“ übersetzte er als „Lasst das Boot nicht aus den Augen!“”. Hier sind der türkische Phraseologismus ,göz kulak olmak“ und seine übliche wörtliche Übertragung ,ganz Auge und Ohr sein" im Deutschen falsche Freunde. Der türkische Phraseologismus „göz kulak olmak" bedeutet im Allgemeinen 'beobachten', aber der deutsche Phraseologismus „ganz Auge und Ohr sein“ hat die Bedeutung „genau aufpassen“ (Alkok 2007: 116). Indem er in den Sinn des Werks eindrang, seine tiefgreifende Sprachkompetenz bewies und gleich oder ähnlich klingende Wörter beachtete, beseitigte er mögliche Übersetzungsfehler.

\section{Schlussbetrachtung}

Wie in der vorliegenden Arbeit betont wurde, ist die literarische Übersetzung eine Sprach- und Kulturarbeit. Daher reicht es nicht aus, ausschließlich die Standartsprache zu beherrschen. Zudem setzt sie u.a. auch kulturelle, geschichtliche, gesellschaftliche, politische und wirtschaftliche Kenntnisse voraus.

Bischoff als ein Kind, dessen Familie in der republikanischen Zeit der Türkei aus Deutschland floh und Zuflucht in der Türkei fand, verbrachte einen Teil seiner 
Kindheit und der Jugendzeit in der Türkei. In der damaligen Zeit lernte und erfasste er die türkische Sprache und Kultur. Diesen gezwungenen Aufenthalt verwandelte er später zu einem Vorteil. Er fing an, die Bücher der türkischen Literatur ins Deutsche zu übersetzen. Die Jahre, die er als ein „Haymatloser“ in der Türkei verbrachte und die Erfahrungen, die er sammelte brachten ihm sowohl im Hinblick auf die Sprache als auch auf die Kultur Nutzen zum Übersetzen. Es war ihm somit gelungen, als Kulturträger zwischen dem Türkischen und Deutschen bekannt zu werden. Das Hauptziel Bischoffs beim Übersetzen war es, die deutsche und türkische Sprache und Kultur zusammenzubringen und dass der deutsche Leser die türkische Kultur und Sprache bzw. die Türkei richtig und gründlich kennenlernt.

Neben der Beherrschung der türkischen Standartsprache und Kultur kannte Bischoff auch deren Schreibregeln und Sprachgebrauch, auch die Dialekte und die Umgangssprache bzw. das anatolische Türkisch. Dadurch diente alles dazu, was Bischoff in der Türkei erlebte, die literarischen Werke der türkischen Literatur ins Deutsche äquivalenter zu übersetzen. Bei ihm waren die Ausgangs- und Zielsprache verschachtelt. Er harmonsierte die türkische und deutsche Kultur. Durch seine sprachlichen und kulturellen Erfahrungen reduzierte er die Interferenzfehler soweit wie möglich. Sein Fenster öffnete sich zu zwei Welten, zum Deutschen und zum Türkischen. Auffällig ist aber, dass er beide als eins akzeptierte.

Die Übersetzungstechnik Bischoffs war die reproduzierende, was seine Kreativität zeigte. Er erfasste und interpretierte die türkische Sprache und Kultur als ein Deutschstämmiger, der lange in der Türkei lebte, aus einem fremden und einheimischen Blick. In dieser Hinsicht konnte er literarische Texte im Rahmen der Form und des Inhalts tiefgreifend analysieren, interpretieren und dann übersetzen. Er war aufgrund der Möglichkeit, das Allgemeine und das Besondere des Türkischen in der Türkei erfahren zu können, privilegiert.

\section{Literaturverzeichnis}

Alkok, Pervin (2007): Phraseologismen als Übersetzungsproblem. Eine türkisch-deutsche Analyse auf der Grundlage von Yaşar Kemals Werk „Firat Suyu Kann Aklyor Baksana“ und Cornelius Bischoffs deutsche Übersetzung „Die Ameiseninsel“. Magisterarbeit. Mersin Universität. Institut für Sozialwissenschaften. Mersin.

Arslan, Müge (2013): Die Lexeme „Heimatlos" und „Heimatlosigkeit" im Deutschen und im Türkischen. Hacettepe Universität Institut für Sozialwissenschaften, Magisterarbeit. Ankara.

Balcı, Tahir (2015): Yaşar Kemal'in "Kuşlar da Gitti" Romanı ve Almanca Çevirisindeki Renk Sözcükleri. Yaşar Kemal Çukurova Ödülü 2015. In: Duyuler, D., Ed., Ankara: Öz Baran Ofset. 223-232.

Bartsch, Patrick (1999: Kritik der deutschen Übersetzungen von Yaşar Kemals Ince Memed. Magisterarbeit, Türkologie. Otto Friedrich Universität, Bamberg. 
Bischoff, Cornelius (2010): Dilimin Sınırları. Değişen Sosyal Hayat ve Çeviri Dili Türkçe. In: Pazarkaya, İ. (Hg.). Dilin Çağrısı. Yüksek Pazarkaya Ellinci Sanat Yılında Armă̆an. İstanbul: Tüyap. 169-174.

Bischoff, Cornelius (1996): Vortrag zum Thema: Nachkommen der Exilanten als Brücke zwischen den Völkern. In: Tömer Çeviri Dergisi. Sayı 9/Güz 96. 44-48.

Bischoff, Cornelius (1989): Probleme bei der Übersetzung türkischer Partizipien. In: ÜbersetzerWorkshop. 22-24. November 1988 im Kulturinstitut. Dosttan Dosta Kitap, Dialog durch Literatur. 35-41.

Bozay, Kemal (2001): Exil Türkei. Ein Forschungsbeitrag zur deutschsprachigen Emigration in der Türkei (1933-1945). Münster: LIT Verlag.

Çiftlikçi, Ramazan (1993): Yaşar Kemal. Yazar-Eser-Uslüp. Doktora Tezi. İnönü Üniversitesi Sosyal Bilimler Enstitüsü Türk Dili ve Edebiyatı Eğitimi Anabilim Dalı. Malatya.

Dağyeli-Bohne, Helga (1989): Verhältnis Autor-Lektor-Übersetzer. Übersetzungsprobleme hinsichtlich der Zeiten und Metaphern im Türkischen und Deutschen. In: Übersetzer-Workshop. 22-24 November 1988, im Deutschen Kulturinstitut. Dosttan Dosta Kitap: Ankara. 43-53.

Dikici, Christine (2015): Die Rezeption der türkischen Literatur im deutschen Sprachraum unter besonderer Berücksichtigung aktüeller Übersetzungsvorhaben. Doktorarbeit. Sakarya Üniversitesi Sosyal Bilimler Enstitüsü. Sakarya.

Dizdar, Dilek (2014): Auf der Suche nach Trüffelschweinen oder: Übersetzen als Entdecken. In: Kelletat, A./ Tashinsky, A. (Hg.): Übersetzer als Entdecker. Ihr Leben und Werk als Gegenstand translationswissenschaftlicher Forschung. Berlin: Frank\&Timme Verlag. 31-50.

Kemal, Yaşar (1986): Das Unsterblichkeistkraut. Aus dem Türkischen von Cornelius Bischoff. Unionsverlag: Zurich.

Kemal, Yaşar (1999): Ölmez Otu. Dağın Öte Yüzü 3. Istanbul: Adam Yayınları.

Kemal, Yaşar (2001): Ameiseninsel. Übersetzt von Cornelius Bischoff. Zurich: Unionsverlag.

Kemal, Yaşar (2004): Fırat Suyu Kan Akıyor Baksana. İstanbul: Yapı Kredi Yayınları.

Koller, Werner (2011): Einführung in die Übersetzungswissenschaft. 8. Auflage. Tübingen: UTB Verlag.

Kula, Onur Bilge (2016): Yaşar Kemal Romanı: Epik Saflık ve İnsancılın Yazınsallaştırımı.16- 17 Aralık 2016. Yaşar Kemal Sempozyumu. In: Andaç, F. (Ed.). Bir Edebiyat Adası: Yaşar Kemal Seтроzуити. Bursa Nilüfer Belediyesi Rota Ofset. 63-83.

Levý, Jiř́ (2012): Die literarische Übersetzung: Theorie einer Kunstgattung (Auszug) (1963, 1969) / A Tradução Literária - Teoria de um Gênero Artístico (Excerto). Übersetzt von Filipe Neckel, Marcelo Rondinelli. In: Scientia Traductionis 11. S. 24-60.

Möckelmann, Reiner (Hg.) (2004): YAŞAR KEMAL im Gespräch mit seinem Übersetzer CORNELIUS BISCHOFF und interessiertem Publikum im Deutschen Generalkonsulat am 22. Januar 2004. Broschüre des Generalkonsulats der Bundesrepublik Deutschland in Istanbul. https://tuerkei.diplo.de/blob/1563568/3bc059a3a0a9dde3724584ecd0b70966/datei-yasar-kemaldata.pdf (letzter Zugriff: 29.07.2020).

Nişanyan, Sevan (2017): Sözlerin Soy Ă̆acı: Çăgdaş Türkçenin Etimolojik Sözlüğü. http://www.nisanyansozluk.com/ (letzter Zugriff: 11.05.2020).

Öncü, Mehmet Tahir (2019): Türkische Literatur im deutschsprachigen Raum: Ein Überblick. In: Akyıldız, Ercan, Cemile/ Balcı, Yasemin/ Öztürk, Ali Osman: Ex Oriente Lux: West-östlicher Kulturtransfer. Berlin: Logos Verlag. 157-174. 
Öner, Çetin (1993): Gülibik der Hahn. Übersetzt von Cornelius Bischoff. Mülheim an der Ruhr: Verlag an der Ruhr.

Probst, Maximilian (2009): Exil in der Türkei, Baden im Bosporus. https://taz.de/Exil-in-derTuerkei/!5169120/ (letzter Zugriff: 25.01.2020).

Programm von Buchmesse RUHR file://C:/Users/M\%C3\%BCge/Downloads/Programmbroschuere.pdf (letzter Zugriff: 29.04.2020).

Reiß, Katharina (1971): Möglichkeiten und Grenzen der Übersetzungskritik. Kategorien und Kriterien für eine sachgerechte Beurteilung von Übersetzungen. München: Max Hueber Verlag.

Şenocak, Zafer (1989): Die Rolle der literarischen Übersetzung im Kulturaustausch (Einige kritische Gedanken beim Lesen von literarischen Übersetzungen, die von Orientalisten angefertigt wurden). In: Übersetzer-Workshop. 22-24 November 1988, im Deutschen Kulturinstitut. Dosttan Dosta Kitap: Ankara. 55-61.

Tonkul, Hatice (2018): Biogramm von Cornelius Bischoff. https://translex.ege.edu.tr/tr6236/cornelius_bischoff.html (letzter Zugriff: 03.05.2020).

Türk Dil Kurumu [TDK] (2019): Genel Türkçe Sözlük. https://sozluk.gov.tr/ (letzter Zugriff: 11.05.2020).

Unionsverlag (2020): Übersetzungen von Cornelius Bischoff. http://www.unionsverlag.com/info/person.asp?pers_id=1320 (letzter Zugriff: 21.08.2020).

Uyanık, Ayşe (2019): Übersetzungsunterricht durch Textanalyse. Paradigma Akademi: Istanbul.

Uyanık, Ayşe/ Uslu, Zeki (2013): Almanca Ö̆gretiminde Yazınsal Metin Çevirisinin Yeri. Electronic Turkish Studies 8-4. 1407-1422.

Vermeer, Hans J. (2014): Nase-Weise Bemerkungen zum literarischen Übersetzen. In: Kelletat, A. und Tashinskiy, A. (Hg.) (2014). Übersetzer als Entdecker. Ihr Leben und Werk als Gegenstand translationswissenschaftlicher Forschung. Berlin: Frank\&Timme Verlag. 27-30.

Yalçın, Kemal (2011): Haymatlos: Dünya Bizim Vatanımız. Istanbul: Türkiye Iş Bankası Yayınları. 